Pacific Journal of Mathematics

THE LEFSCHETZ NUMBER AND BORSUK-ULAM THEOREMS 


\section{THE LEFSCHETZ NUMBER AND BORSUK-ULAM THEOREMS}

\section{Daniel Henry GotTlieb}

Let $M$ be a manifold, with or without boundary, which is dominated by a finite complex. Let $G$ be a finite group which acts faithfully and freely on $M$. Let $f: M \rightarrow M$ be a $G$-map. Let $\Lambda_{f}$ denote the Lefschetz number of $f$ and let $o(G)$ denote the order of $G$. The main result states, under the conditions above, that $o(G)$ divides $\Lambda_{f}$. Even in the case of compact $M$ this result was not widely known. We use Wall's finiteness obstruction theory to extend the result from compact $M$ to finitely dominated $M$.

The remainder of the paper is devoted to various easy applications of the result. In Theorem 5 we assume that $\pi_{i}(M)$ is finitely generated for all $i>1$. Then we show that if $\pi_{1}(M)$ has torsion, $\pi_{*}(M)$ cannot be only torsion.

In Theorem 6, we have a connected Lie group $L$ acting on $M$ and $f$ is an $L$-map. We show that the orbit map $\omega: L \rightarrow M$ induces the trivial homomorphism on fundamental groups if $\Lambda_{f} \neq 0$. This implies that the action of $L$ on $M$ can be lifted to any regular covering space.

We show that any linear transformation $T: R^{n} \rightarrow R^{n}$ which commutes with the based free action of a finite group $G$ of order greater than 2 must have a non-negative determinant (Theorem 8).

Then we come to the Borsuk-Ulam type results. We consider maps $f:\left(\boldsymbol{C}^{n+1}-0\right) \rightarrow \boldsymbol{C}^{n}$. A primitive $k$-root of unity $\xi$ gives rise to a free $\boldsymbol{Z}_{k}$-action on $\boldsymbol{C}^{n}$. We show that the equation $\sum_{\imath=0}^{k-1} \bar{\xi}^{i} f\left(\xi^{i} x\right)=0$ always has a solution $x \in C^{n+1}-0$. This result gives various conditions on the degeneracy of the images of the orbit of the $Z_{k}$ action in $C^{n}$. In particular, we show that if $f: S^{n} \rightarrow R^{r}$ and if $n \geqq r(p-1)$, then some orbit of the $\boldsymbol{Z}_{p}$-action must be mapped into a point. The proof uses the equation above and Vandermonde determinants.

2. Free actions and the Lefschetz number. A manifold $M$ (or space) is dominated by a finite complex $K$ if there exists maps $f: M \rightarrow K$ and $g: K \rightarrow M$ such that $g \cdot f$ is homotopic to the identity of $M$. We will need various facts about finitely dominated spaces in order to prove the result that $o(G)$ divides $\Lambda_{f}$ for noncompact $M$. It is easily shown that this is true for compact $M$. We use the theory of C.T.C. Wall, [8], to extend to the noncompact case.

Lemma 1. Let $M$ be a finitely dominated manifold. The orbit 
space $M / G$ is a finitely dominated manifold.

Proof. Consider the universal principal $G$-bundle $G \rightarrow E_{G} \rightarrow B_{G}$. Since $G$ acts on $M$, we may replace the fibre to obtain the bundle $M \rightarrow M_{G} \rightarrow B_{G}$. Since $G$ acts freely on $M$, we know that $M_{G}$ is a homotopy equivalent to $M / G$. Since $G$ is finite and acts freely on $M$, we see that $M \rightarrow M / G$ is a covering, so $M / G$ is a manifold, and hence it is finite dimensional.

We know from Wall's work [8] that a space is finitely dominated if and only if it is homotopy equivalent to a finite dimensional $C W$ complex and also homotopy equivalent to a possibly different $C W$ complex of finite type (i.e., a complex whose $n$-skeletons are finite complexes for all $n$ ). Thus $M$ is homotopy equivalent to a complex of finite type. Since $M_{G}$ is the total space of a fibration whose base and fibre are of finite type, $M_{G}$ is homotopy equivalent to a complex of finite type [4; Lemma 1.1]. Also, by the first paragraph, $M_{G}$ is homotopy equivalent to $M / G$ which is a finite dimensional complex. So $M / G$ is finitely dominated.

REMARK. The above lemma follows from Lemma 5.5 in [2].

Now we come to the main result.

THEOREM 2. Let $M$ be a finitely dominated manifold, and let $G$ act freely and faithfully on $M$ and $f: M \rightarrow M$ be a G-map. Then $o(G)$ divides $\Lambda_{f}$.

Proof. First we show the result for a compact manifold $M$ with a finite group $G$ acting freely on $M$ and a $G$-map $f: M \rightarrow M$. We obtain a commutative diagram

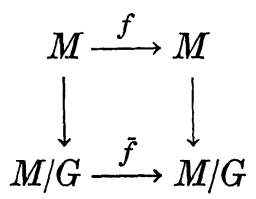

where the vertical maps are covering projections and $\bar{f}$ is induced by $f$. Now $M / G$ is a finite $C W$-complex and we may adjust $\bar{f}$ by a homotopy so that it has finitely many isolated fixed points. Now $f$ restricted to a fibre over a fixed point maps the fibre onto itself and it either leaves all the points fixed or leaves no points fixed. Thus if $x$ is a fixed point of $f$ then all the points in the fibre, which is an orbit of $G$, are fixed, and they all must have the same fixed point index. The fibre contains $o(G)$ points, so $o(G)$ must divide $\Lambda_{f}$. 
Now suppose that $M$ is a finitely dominated manifold. It follows from Wall's theory [8] that the cartesian product of $M$ with a finite complex, whose Euler-Poincare number is zero, must be a homotopy equivalent to a finite complex. Thus we have a compact manifold $K$ and homotopy equivalences $h: K \rightarrow S^{3} \times(M / G)$ and $h^{-1}: S^{3} \times(M / G) \rightarrow K$. We let $G$ act on $S^{3} \times M$ by letting $g(s, m)=$ $(s, g(m))$. Then $G$ acts freely on $S^{3} \times M$ and gives rise to a diagram

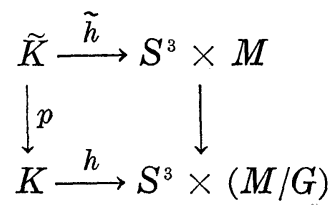

where $\widetilde{K} \rightarrow K$ is in the pull back of the $G$-bundle $S^{3} \times M \rightarrow S^{3} \times$ $(M / G)$. So $\widetilde{K} \rightarrow K$ is a covering space and $\widetilde{h}$ is a $G$-map. Also there is some lifting $\widetilde{h}^{-1}$ of $h^{-1}$ which is a $G$-map and $\tilde{h} \circ \widetilde{h}^{-1}$ is homotopic to the identity by equivariant homotopies.

Now let $F: S^{3} \times M \rightarrow S^{3} \times M$ be given by $F(s, m)=\left(s_{0}, f(m)\right)$ where $s_{0} \in S^{3}$ is a base point. $F$ is a $G$-map and so the composition $\tilde{h}^{-1} F \tilde{h}$ : $\widetilde{K} \rightarrow K$ is a $G$-map. Now $\Lambda_{\tilde{h}-1}^{-1} \tilde{h}=\Lambda_{F}=\Lambda_{f}$ and by the theorem for compact complexes $o(G)$ divides $\Lambda_{\tilde{h}^{-}{ }_{F \tilde{h}}}$ and hence it divides $\Lambda_{f}$.

REMARK. An earlier version of this theorem was proved in [1; Theorem 4] by transfer methods for $G$ a cyclic group and $M$ homotopy equivalent to a finite complex. Nakaoka in [7] developed a transfer based on the coincidence number $\Lambda_{f, g}$ for maps $f$ and $g$ from $M$ to itself where $M$ is a compact manifold. Using a result related to these transfers he succeeded in showing that $o(G)$ divides $\Lambda_{f, g}$ where $f$ and $g$ are $G$-maps. This result gives Theorem 2 in the compact case. The compact case was originally proved by G. Hirsch [3a].

Corollary 3. o $(G)$ divides $\chi(M)$, the Euler-Poincare number.

CoRollary 4. If $f: M \rightarrow M$ is a G-map where $G$ acts freely and is finite, then $f$ cannot be homotopic to a constant map.

3. The fundamental group. At this time, despite our great knowledge of homotopy groups, we do not possess a single example of a finite complex, with nontrivial higher homotopy groups, whose homotopy groups are completely known. The following result illustrates a difficulty. It generalizes the famous result that if $\pi$ has torsion, then $K(\pi, 1)$ cannot be a finite complex. 
THEOREM 5. Assume that $M$ is a manifold such that $\pi_{i}(M)$ is finitely generated for all $i>1$. Then if some nontrivial element of $\pi_{1}(M)$ has finite order, some element of $\pi_{i}(M)$ does not have finite order for some $i>1$. (Here we need not assume that $M$ has finite type.)

Proof. Consider the universal cover $\tilde{M}$ of $M$. Now $\tilde{M}$ has finite dimension and $\pi_{i}(\widetilde{M}) \cong \pi_{i}(M)$ is finitely generated for $i>1$, and $\pi_{1}(\tilde{M})=0$. Hence it follows from Wall's theory that $\widetilde{M}$ is homotopy equivalent to a finite complex. Now $\pi_{1}(M)$ acts freely on $\widetilde{M}$ and if it contains an element of finite order, then a finite cyclic group $G$ acts freely on $\tilde{M}$ and $o(G)$ divides $\chi(\tilde{M})$ by Corollary 3 .

Now if $\pi_{i}(M) \cong \pi_{i}(\tilde{M})$ were all torsion groups for $i>1$, then by the Hurewicz theorem mod finite groups, the homology groups of $H_{i}(\tilde{M})$ would be finite for all $i$. Thus $\chi(\widetilde{M})=1$ so $o(G)=1$ which contradicts the hypothesis that $G$ is not trivial.

Now we turn to the action of a Lie group $L$ on a compact manifold $M$ with or without boundary. There is the orbit map or evaluation map $\omega: L \rightarrow M$ given by evaluating $l \in L$ at a base point $m_{0} \in M$. Thus $\omega: l \mapsto l\left(m_{0}\right)$. We are concerned with the induced homomorphism $\omega_{*}: \pi_{1}(L) \rightarrow \pi_{1}(M)$.

THEOREM 6. Let $L$ be a connected Lie group acting on a compact manifold $M$ with or without boundary. Suppose there is an equivariant map $f: M \rightarrow M$ such that $\Lambda_{f} \neq 0$. Then $\omega_{*}: \pi_{1}(L) \rightarrow \pi_{1}(M)$ is trivial.

Proof. Every element $\alpha \in \pi_{1}(L)$ can be represented by a homomorphism $S^{1} \rightarrow L$. Thus we need only prove the theorem in the case of $L=S^{1}$.

We shall show that the $S^{1}$ action must have a fixed point and this will give us $\omega_{*}=0$. There is an element $t \in S^{1}$ such that $\left\{t^{n} \mid\right.$ all $\left.n \in Z\right\}$ is dense in $S^{1}$. There is a sequence $s_{p} \in S^{1}$ for $p$ a prime such that $s_{p}$ converges to $t$ and $s_{p}$ has order $p$. Since only a finite number of primes divide $\Lambda_{f}$, all but a finite number of $s_{p}$ must have a fixed point $x_{p}$ by Theorem 2. Now the sequence $\left\{x_{p}\right\}$ must have a cluster point $x$ since $M$ is compact, thus $x$ must be a fixed point for $t$ and hence a fixed point for all of $S^{1}$.

COROLLARY 7. With the hypotheses above, the action of $L$ on $M$ can be lifted to any regular covering $\tilde{M}$ of $M$.

Proof. By Corollary 11 of [3], $\omega_{*}=0$ is sufficient. 
REMARK. Theorem 6 is related to Theorem 5 of [1]. Using the first paragraph of the proof of Theorem 6 and Theorem 5 of [1] we can eliminate the compactness condition on $M$. Theorem 6 and the result from [1] that $\omega_{*}: \widetilde{H}_{*}(L) \rightarrow \widetilde{H}_{*}(M)$ is zero for integral homology under the hypothesis of Theorem 6 are interesting to compare.

4. Borsuk-Ulam theorems. In this section we study spheres and complex and real vector spaces. The classical Borsuk-Ulam theorem involves a continuous map $f:\left(R^{n+1}-0\right) \rightarrow R^{n}$ and states that some orbit of the action $x \mapsto-x$ on $R^{n+1}-0$ is mapped onto a single point of $R^{n}$. We investigate the situation of a map $\left(\boldsymbol{C}^{n+1}-\right.$ $0) \stackrel{f}{\rightarrow} C^{n}$ and ask if an orbit of the action $x \mapsto \xi x$ is mapped onto a single point.

But before we begin with the Borsuk-Ulam theorems we prove a different result on the determinant of a linear transformation. We say that a finite group $G$ acts based freely on $R^{n}$ if it acts faithfully on $R^{n}$ and freely on $R^{n}-0$. We say a linear transformation $T: R^{n} \rightarrow R^{n}$ commutes with the action of $G$ if $T$ commutes with every element $g \in G$.

THEOREM 8. If $G$ acts based freely on $R^{n}$ and if $T$ is a linear transformation which commutes with the action of $G$, then $\operatorname{det} T \geqq 0$ or $o(G)=1$ or 2 .

Proof. If $\operatorname{det} T \neq 0$, then $T$ is an isomorphism and we can regard $T: R^{n}-0 \rightarrow R^{n}-0$. Now $\Lambda_{T}=1+(-1)^{n-1} \operatorname{deg} T$ and $\operatorname{deg} T=$ $\operatorname{det} T /|\operatorname{det} T|$. Assume $o(G)>2$. Then $n$ must be even since otherwise $\chi\left(R^{n}-0\right)=2$ and $o(G)$ divides $\chi\left(R^{n}-0\right)$.

Thus $\Lambda_{T}=1-(\operatorname{det} T /|\operatorname{det} T|)$ and so $\Lambda_{T}$ can either be 2 or 0 . But it cannot be 2 since $o(G)>2$ and divides $\Lambda_{T}$. Then $\Lambda_{T}=0$ and hence $\operatorname{det} T>0$.

Now we come to the main underlying result for the BorsukUlam theorem.

THEOREM 9. Let $G$ be a finite group acting based freely on a vector space $V$ and let $W$ be a proper invariant subspace. Then any G-map $f:(V-0) \rightarrow W$ must contain 0 in its image.

Proof. Suppose 0 is not in the image of $f$. Then the composition $(V-0) \rightarrow W-0 \hookrightarrow V-0$ is a $G$-map and it must be homotopic to a constant, so by Corollary 4 either $G$ is trivial or $f(x)=0$ for some $x \in V-0$. 
THEOREM 10. Let $\xi$ be a primitive kth root of unity. Let $f: \boldsymbol{C}^{n+1}-0 \rightarrow C^{n}$ be any continuous map. Then there is an $x \in C^{n}-$ 0 such that $\sum_{i=0}^{k-1} \bar{\xi}^{i} f\left(\xi^{i} x\right)=0$.

Proof. This follows from Theorem 9 after noting that $x \mapsto \xi x$ leads to a based free action of $\boldsymbol{Z}_{k}$ on $\boldsymbol{C}^{n+1}-0$ and that $\boldsymbol{C}^{n}$ can be regarded as an invariant subspace and $F(x)=\sum_{i=1}^{k} \bar{\xi} f\left(\xi^{i} x\right)$ is an equivariant map.

Observe that we may let $S^{2 n+1}$ be the unit sphere in $C^{n+1}$ and Theorem 10 guarantees that $\sum_{i=1}^{k} \bar{\xi}^{i} f\left(\xi^{i} x\right)=0$ for some $x \in S^{2 n+1}$ and $f: S^{2 n+1} \rightarrow C^{n}$.

We will call the set $\left\{x, \xi x, \cdots, \xi^{k-1} x\right\}$ a $k$-orbit on $S^{2 n+1}$ where $\xi$ is a primative $k$ th root of unity.

CoRollary 11. Given $k$ and $f: S^{2 n+1} \rightarrow C^{n}$, there is a k-orbit whose image lies in a $k-2$ dimensional complex hyperplane.

Proof. Choose $x$ so that $\sum \bar{\xi}^{i} f\left(\xi^{i} x\right)=0$. Let $x_{i}=f\left(\xi^{i} x\right)$. Consider the set of $k-1$ vectors. $\left\{\left(x_{1}-x_{k}\right), \cdots,\left(x_{k-1}-x_{k}\right)\right\}$. These lie in a $k-2$ dimensional subspace since they are linearly dependent since they satisfy $\sum_{i=1}^{k} \bar{\xi}^{i}\left(x_{i}-x_{k}\right)=0$. Hence the translation by $x_{k}$ of this subspace is a $k-2$ dimensional hyperplane which contains the vectors $x_{1}, \cdots, x_{k}$.

Corollary 12. Given $k$ and $f: S^{2 n+1} \rightarrow \boldsymbol{R}^{n}$, there is a k-orbit whose image under $f$ lies in a $k-3$ dimensional real hyperplane.

Proof. The equation $\sum_{i=1}^{k} \bar{\xi}^{i} f\left(\xi^{i} x\right)=0$ gives two equations, one for the real part and one for the imaginary part.

Corollary 13. A map $f: S^{2 n+1} \rightarrow R^{n}$ carries some 3-orbit to a point.

CoRollary 14. A map $f: S^{2 n+1} \rightarrow \boldsymbol{R}^{n}$ carries some 4-orbit into one or two points so the two pairs of antipodal points are each carried into a point.

Proof. Let $x_{j}=f\left(\xi^{j} x\right)$. Then $-i x_{1}-x_{2}+i x_{3}+x_{4}=0$.

So $x_{1}=x_{3}$ and $x_{2}=x_{4}$.

THEOREM 15. Suppose $p$ is a prime and $f: S^{n} \rightarrow R^{r}$. If $n \geqq$ $r(p-1)$, then there is a p-orbit whose image is a single point. 
Proof. For $p=2$ this is the classical Borsuk-Ulam theorem. For $p>2$ we see that $n$ must be odd and $r(p-1)$ must be even so we may assume that $n>r(p-1)$.

Let $n=2 k-1$. Then we extend $f: S^{2 k-1} \rightarrow R^{r}$ radially to a map $g: C^{k} \rightarrow R^{r}$ by letting $g(z)=\|z\| f(z /\|z\|)$. Then we double the dimension of the range and domain and define a map $G: C^{2 k} \rightarrow C^{r}$ by setting $G\left(z_{1}, z_{2}\right)=g\left(z_{1}\right)+i g\left(z_{2}\right)$ where $\left(z_{1}, z_{2}\right) \in C^{k} \oplus \boldsymbol{C}^{k} \cong \boldsymbol{C}^{2 k}$. Now we define a map $F:\left(C^{2 k}-0\right) \rightarrow C^{r(p-1)}$ by letting $F(z)=\left(G(z), G(z)^{2}, \cdots\right.$, $\left.G(z)^{p-1}\right)$ where we understand that a vector $v=\left(v_{1}, \cdots, v_{r}\right.$ ) raised to the $j$ th power is the vector $v^{j}=\left(v_{1}^{j}, \cdots, v_{r}^{j}\right)$.

Now since $2 k>r(p-1)$ (because $n \geqq r(p-1)$ by hypothesis), we can apply Theorem 10 which guarantees a solution to the equation

$$
\sum_{j=0}^{p-1} \bar{\xi}^{j} F\left(\xi^{j} x\right)=0
$$

Let $x_{j}=G\left(\xi^{j} x\right)$ and $x_{j}^{k}=\left(G\left(\xi^{j} x\right)\right)^{k}$. Then we obtain the vector equations

$$
\begin{gathered}
\sum_{j=0}^{p-1} \bar{\xi}^{j} x_{j}=0 \\
\sum_{j=0}^{p-1} \bar{\xi}^{j} x_{j}^{2}=0 \\
\vdots \\
\sum_{j=0}^{p-1} \bar{\xi}^{j} x_{j}^{p-1}=0 .
\end{gathered}
$$

These equations are vector equations since $x_{j}^{k}$ is a vector. If we look at, say, the first coordinates of the vectors $\left\{x_{j}^{k}\right\}$ and use $x_{j}^{k}$ to denote its own first coordinate then the set of equations can be written in matrix form as

$$
\left(\begin{array}{cccc}
1 & 1 & \cdots & 1 \\
x_{1} & x_{2} & \cdots & x_{p-1} \\
& & \vdots & \\
x_{1}^{p-1} & & \cdots & x_{p-1}^{p-1}
\end{array}\right)\left(\begin{array}{c}
1 \\
\bar{\xi} \\
\vdots \\
\bar{\xi}^{p-1}
\end{array}\right)=\left(\begin{array}{c}
0 \\
0 \\
\vdots \\
0
\end{array}\right) .
$$

Now the matrix gives the Vandermonde matrix and

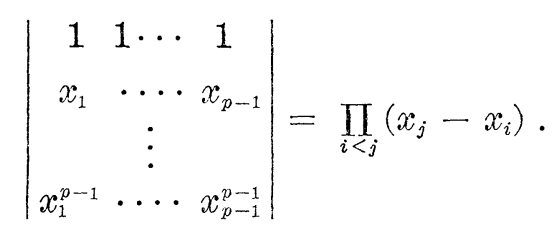

Then an easy induction argument shows that the row echelon form of the Vandermonde matrix consists of rows composed of entries which are 0's or 1's. We have the equation 


$$
\left(\begin{array}{cccccc}
1 & 0 & 0 & 1 & 0 & 0 \\
& & \vdots & & \\
& & \vdots & &
\end{array}\right)\left(\begin{array}{c}
1 \\
\bar{\xi} \\
\vdots \\
\bar{\xi}^{p-1}
\end{array}\right)=\left(\begin{array}{c}
0 \\
0 \\
\vdots \\
0
\end{array}\right)
$$

Since $p$ is a prime and $\xi$ is a primitive $p$ th root of unity the only sum of these roots which equals zero is the sum of them all, $1+$ $\xi+\cdots+\xi^{p-1}=0$. Thus the row echelon form must be

$$
\left(\begin{array}{llll}
1 & 1 & \cdots & 1 \\
0 & 0 & \cdots & 0 \\
& \vdots & & \\
0 & \cdots & 0 & 0
\end{array}\right)
$$

which can only happen if $x_{1}=x_{2}=\cdots=x_{p-1}$. Thus all the 1 st coordinates of the vectors $x_{1}, \cdots, x_{p-1}$ agree, and similarly all the $j$ th coordinates agree. So

$$
G(x)=G(\xi x)=\cdots=G\left(\xi^{p-1} x\right) \text { for some } x \in C^{2 k}-0 .
$$

This implies that

$$
g(z)=g(\xi z)=\cdots=g\left(\xi^{p-1} z\right)
$$

for some $z \in C^{k}-0$ and since $g$ is a radial extension of $f$ we see that $f$ maps an orbit to a single point.

Remark. Theorem 15 was proved by Munkholm [5] and Nakaoka [6]. In both cases their methods apply in a more general situation. On the other hand Corollary 14 is new and is different from Mumkholm's and Nakaoka's work in that it considers an action of a cyclic group of order 4 and 4 is not a prime.

\section{REFERENCES}

1. A. Casson and D. H. Gottlieb, Fibrations with compact fibres, Amer. J. Math., 99, (1977), 159-189.

2. A. L. Edmonds, Equivariant regular neighborhoods in transformation groups, Proc. of the Conference at the University of Newcastle upon Tyne, August 1976, Editor Czes Kosniowski, Cambridge University Press, 51-69.

3. D. H. Gottlieb, Lifting actions in fibrations, Geometric applications of homotopy theory I, (Proceedings of the Evanston Conference, 1977.) Lecture Notes in Mathematics $\# 657$, Springer-Verlag, 217-254.

3a. G. Hirsch, Ann. Sci. Ecole. Sup. (3), 60 (1943), 113-142.

4. G. Mislin, Conditions for finite domination for certain complexes, Proceedings of the Vancouver Conference in Algebraic topology, 219-224, Lecture Notes in Mathematics \#673, Springer-Verlag. 
5. H. J. Munkholm, Borsuk-Ulam type theorems for proper $\boldsymbol{Z}_{p}$-actions on (mod $p$ homology) n-spheres, Math. Scand., 24 (1969), 167-185.

6. M. Nakaoka, Generalizations of the Borsuk-Ulam theorem, Osaka J. Math., 7 (1970), 423-441.

7. Coincidence Lefschetz numbers for a pair of fibre preserving maps,

J. Math. Soc. Japan, 32 (1980), 751-779.

8. C.T.C. Wall, Finiteness conditions for CW-complexes, Ann. of Math., 81 (1965), $56-69$.

Received October 25, 1980 and in revised form May 20, 1981. This research was partially supported by an NSF Grant.

\section{PuRdue University}

West LafayetTe, IN 47907

AND

UNIVERSITY OF CALIFORNIA

LOS ANGELES, CA 90024 



\section{PACIFIC JOURNAL OF MATHEMATICS}

\section{EDITORS}

DONALD BABBITT (Managing Editor)

University of California

Los Angeles, California 90024

Hugo RossI

University of Utah

Salt Lake City, UT 84112

C. C. Moore and Arthur Agus

University of California

Berkeley, CA 94720
J. DugundJI

Department of Mathematics University of Southern California Los Angeles, California 90007

R. FInN and J. Milgram Stanford University Stanford, California 94305

ASSOCIATE EDITORS
R. ARNES
E. F. BeCKenbach
B. H. NeumanN
F. WOLF
K. YoSHIDA

\section{SUPPORTING INSTITUTIONS}

UNIVERSITY OF ARIZONA

UNIVERSITY OF BRITISH COLUMBIA

CALIFORNIA INSTITUTE OF TECHNOLOGY

UNIVERSITY OF CALIFORNIA

MONTANA STATE UNIVERSITY

UNIVERSITY OF NEVADA, RENO

NEW MEXICO STATE UNIVERSITY

OREGON STATE UNIVERSITY
UNIVERSITY OF OREGON

UNIVERSITY OF SOUTHERN CALIFORNIA

STANFORD UNIVERSITY

UNIVERSITY OF HAWAII

UNIVERSITY OF TOKYO

UNIVERSITY OF UTAH

WASHINGTON STATE UNIVERSITY

UNIVERSITY OF WASHINGTON 


\section{Pacific Journal of Mathematics}

\section{Vol. 103, No. $1 \quad$ March, 1982}

Abdul Aziz, On the zeros of composite polynomials ..................

Salomon Benzaquen and Enrique M. Cabaña, The expected measure of the level sets of a regular stationary Gaussian process $\ldots \ldots \ldots \ldots \ldots$

Claudio D’Antoni, Roberto Longo and László Zsidó, A spectral mapping theorem for locally compact groups of operators $\ldots \ldots \ldots \ldots \ldots \ldots \ldots 17$

Ronald Dotzel, Semifree finite group actions on homotopy spheres ........ 25

Daniel H. Gottlieb, The Lefschetz number and Borsuk-Ulam theorems . . . . . 29

Shui-Hung Hou, On property $(Q)$ and other semicontinuity properties of

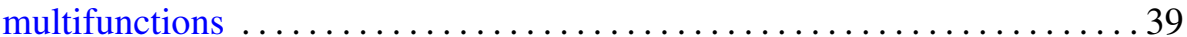

Kevin Mor McCrimmon, Compatible Peirce decompositions of Jordan triple systems

Mitsuru Nakai, Corona problem for Riemann surfaces of Parreau-Widom

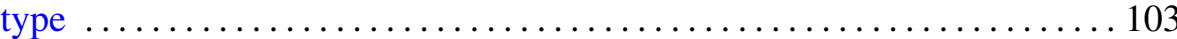

Jack Ray Porter and R. Grant Woods, Extensions of Hausdorff spaces . . . . 111

Milton Rosenberg, Quasi-isometric dilations of operator-valued measures

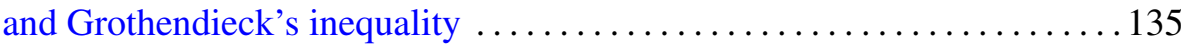

Joseph L. Taylor, A bigger Brauer group $\ldots \ldots \ldots \ldots \ldots \ldots \ldots \ldots \ldots \ldots$

Thomas Vogel, Symmetric unbounded liquid bridges . . . . . . . . . . . 205

Steve Wright, The splitting of operator algebras. II ............... 243 